\title{
Isolation and Characterisation of Alongshan Virus in Russia
}

Ivan S. Kholodilov 1,+ ${ }^{1}$, Alexander G. Litov ${ }^{1,+}$, Alexander S. Klimentov ${ }^{2,3}$, Oxana A. Belova ${ }^{1}$, Alexandra E. Polienko ${ }^{1}$, Nikolai A. Nikitin ${ }^{4}$ (D), Alexey M. Shchetinin ${ }^{5}$, Anna Y. Ivannikova ${ }^{1}$, Lesley Bell-Sakyi ${ }^{6}$, Alexander S. Yakovlev ${ }^{1}$, Sergey V. Bugmyrin ${ }^{7}$, Liubov A. Bespyatova ${ }^{7}$, Larissa V. Gmyl ${ }^{2}$, Svetlana V. Luchinina ${ }^{8}$, Anatoly P. Gmyl ${ }^{1, \ddagger}$, Vladimir A. Gushchin ${ }^{4,5}$ and Galina G. Karganova 1,9,*

1 Laboratory of Biology of Arboviruses, Chumakov Institute of Poliomyelitis and Viral Encephalitides, FSBSI Chumakov FSC R\&D IBP RAS, 108819 Moscow, Russia; ivan-kholodilov@bk.ru (I.S.K.); novosti-wxo@yandex.ru (A.G.L.); mikasusha@bk.ru (O.A.B.); polienko.ae@yandex.ru (A.E.P.); strannotut@gmail.com (A.Y.I.); alex-montreal@yandex.ru (A.S.Y.); apgmyl@mail.ru (A.P.G.)

2 Laboratory of Biochemistry, Chumakov Institute of Poliomyelitis and Viral Encephalitides, FSBSI Chumakov FSC R\&D IBP RAS, 108819 Moscow, Russia; aklimentov@mail.ru (A.S.K.); lvgmyl@mail.ru (L.V.G.)

3 Laboratory of Biology and Indication of Arboviruses, Department Ivanovsky Institute of Virology, Gamaleya Federal Research Centre for Epidemiology and Microbiology, Ministry of Health of the Russian Federation, 123098 Moscow, Russia

4 Faculty of Biology, Lomonosov MSU, 119991 Moscow, Russia; nik.nikitin@gmail.com (N.A.N.); wowaniada@gmail.com (V.A.G.)

5 Pathogenic Microorganisms Variability Laboratory, Gamaleya Federal Research Centre for Epidemiology and Microbiology, Ministry of Health of the Russian Federation, 123098 Moscow, Russia; shchetinin.alexey@yandex.ru

6 Department of Infection Biology, Institute of Infection and Global Health, University of Liverpool, Liverpool L3 5RF, UK; 1.bell-sakyi@liverpool.ac.uk

7 Laboratory for Animal and Plant Parasitology, Institute of Biology of Karelian Research Centre, Russian Academy of Sciences (IB KarRC RAS), 185910 Petrozavodsk, Russia; sbugmyr@mail.ru (S.V.B.); gamasina@mail.ru (L.A.B.)

8 Russian Federal Service for Surveillance on Consumer Rights Protection and Human Wellbeing, 454092 Chelyabinsk, Russia; luchinina_sv@74.rospotrebnadzor.ru

9 Department of Organization and Technology of Immunobiological Preparations, Institute for Translational Medicine and Biotechnology, Sechenov University, 119991 Moscow, Russia

* Correspondence: karganova@bk.ru; Tel.: +7-495-841-9327

+ These authors contributed equally to this article.

$\ddagger$ Deceased.

Received: 23 February 2020; Accepted: 24 March 2020; Published: 26 March 2020

Abstract: In recent decades, many new flavi-like viruses have been discovered predominantly in different invertebrates and, as was recently shown, some of them may cause disease in humans. The Jingmenvirus (JMV) group holds a special place among flaviviruses and flavi-like viruses because they have a segmented ssRNA(+) genome. We detected Alongshan virus (ALSV), which is a representative of the JMV group, in ten pools of adult Ixodes persulcatus ticks collected in two geographically-separated Russian regions. Three of the ten strains were isolated in the tick cell line IRE/CTVM19. One of the strains persisted in the IRE/CTVM19 cells without cytopathic effect for three years. Most ALSV virions purified from tick cells were spherical with a diameter of approximately $40.5 \mathrm{~nm}$. In addition, we found smaller particles of approximately $13.1 \mathrm{~nm}$ in diameter. We obtained full genome sequences of all four segments of two of the isolated ALSV strains, and partial sequences of one segment from the third strain. Phylogenetic analysis on genome segment 2 of the JMV group clustered our novel strains with other ALSV strains. We found evidence for the existence of a novel upstream open reading frame in the glycoprotein-coding segment of ALSV and other members of the JMV group. 
Keywords: Alongshan virus; Jingmen tick virus; open reading frame; flavivirus; flavi-like virus; Ixodes persulcatus; tick cell line

\section{Introduction}

In recent decades, many new flavi-like viruses have been discovered in different invertebrates. All of them share homology in nonstructural proteins with the well-studied flavivirus RNA-dependent RNA polymerase NS5 and RNA helicase-protease NS3, but they differ greatly from each other regarding other proteins, as well as in overall genome size and structure [1].

The Jingmenvirus (JMV) group holds a special place among flavi-like viruses because in contrast to the "classic" flaviviruses and other flavi-like viruses, they have a segmented ssRNA(+) genome [1-5], which is more commonly observed in viruses of fungi and plants [6]. The only other known viruses with a segmented ssRNA(+) genome are representatives of the family Nodaviridae and infect insects (Alphanodavirus) and fish (Betanodavirus) [7].

The JMV group is diverse and includes the Guaico Culex virus and Jingmen tick virus clades [8]. Two segments of the JMV group genome are homologous to the regions of the Flavivirus genome encoding NS3 and NS5 proteins [1,2]. The remaining segments are specific only to JMVs. Moreover, these segments contain open reading frames (ORFs) that have no homology between viruses of the Guaico Culex virus and Jingmen tick virus clades [2]. Additionally, in contrast to the "classic" flaviviruses that have one long open reading frame (ORF) and virion size of 40-60 nm, these viruses have several ORFs within single segments and virion sizes varying from 30-35 $\mathrm{nm}$ to 70-100 $\mathrm{nm}$ [2,4,5]. It has also been suggested that genomic RNAs of a multicomponent virus can be packaged in separate virions [2], although this has not yet been demonstrated.

The geographic distribution of the JMV group is very wide, encompassing Asia $[1,4,9,10]$, Europe [8,10-12], Central and South America [2,3,8,13,14] and Africa [2,8]. Virus RNAs of this group have been detected not only in different species of insects, e.g., mosquitoes [5] and Drosophila melanogaster [15], but also in the nematode Toxocara canis [16,17] and ticks [1,4,10] including Rhipicephalus microplus [8,14], Ixodes ricinus [8,12] and I. persulcatus [5,9]. Jingmen tick virus (JMTV) has been isolated from $R$. microplus ticks with restricted in vitro multiplication in tick and mammalian cells [3,4], and from Amblyomma javanense ticks with continuous cultivation in a tick cell line [9]. Some representatives of the JMV group were detected in patients with other infections transmitted by arthropods [11].

Alongshan virus (ALSV), a representative of the JMV group, was detected in I. persulcatus ticks, Culex tritaeniorhynchus and Anopheles yatsushiroensis mosquitoes in China [5] and later in I. ricinus ticks in Finland and France [8,12]. ALSV was suspected to infect mammals and cause illness in humans $[5,18]$.

Our work presents data on the isolation of ALSV in the Russian Federation and new data on the genome organization of this and other known representatives of the JMV group.

\section{Materials and Methods}

\subsection{Collection and Processing of Ticks}

In 2014, from the beginning of May to the middle of June, unfed nymphal and adult ticks were collected by flagging from vegetation in the Republic of Karelia and Chelyabinsk region (Figure 1). Ticks were identified using taxonomic keys $[19,20]$. Nymphs were homogenized in a pool of 13 individuals and adult ticks were homogenized in pools of 3-5 individuals according to species composition, location, and route of collection using the laboratory homogenizer TissueLyser II (QIAGEN, Germany) in Medium 199 supplemented with Earle's salts (FSBSI Chumakov FSC R\&D IBP RAS, Russia) and antibiotics. All homogenates were tested for the presence of flavivirus RNA using a heminested RT-PCR and primers targeting a conserved region of the flavivirus NS5 gene, as described earlier [21]. 


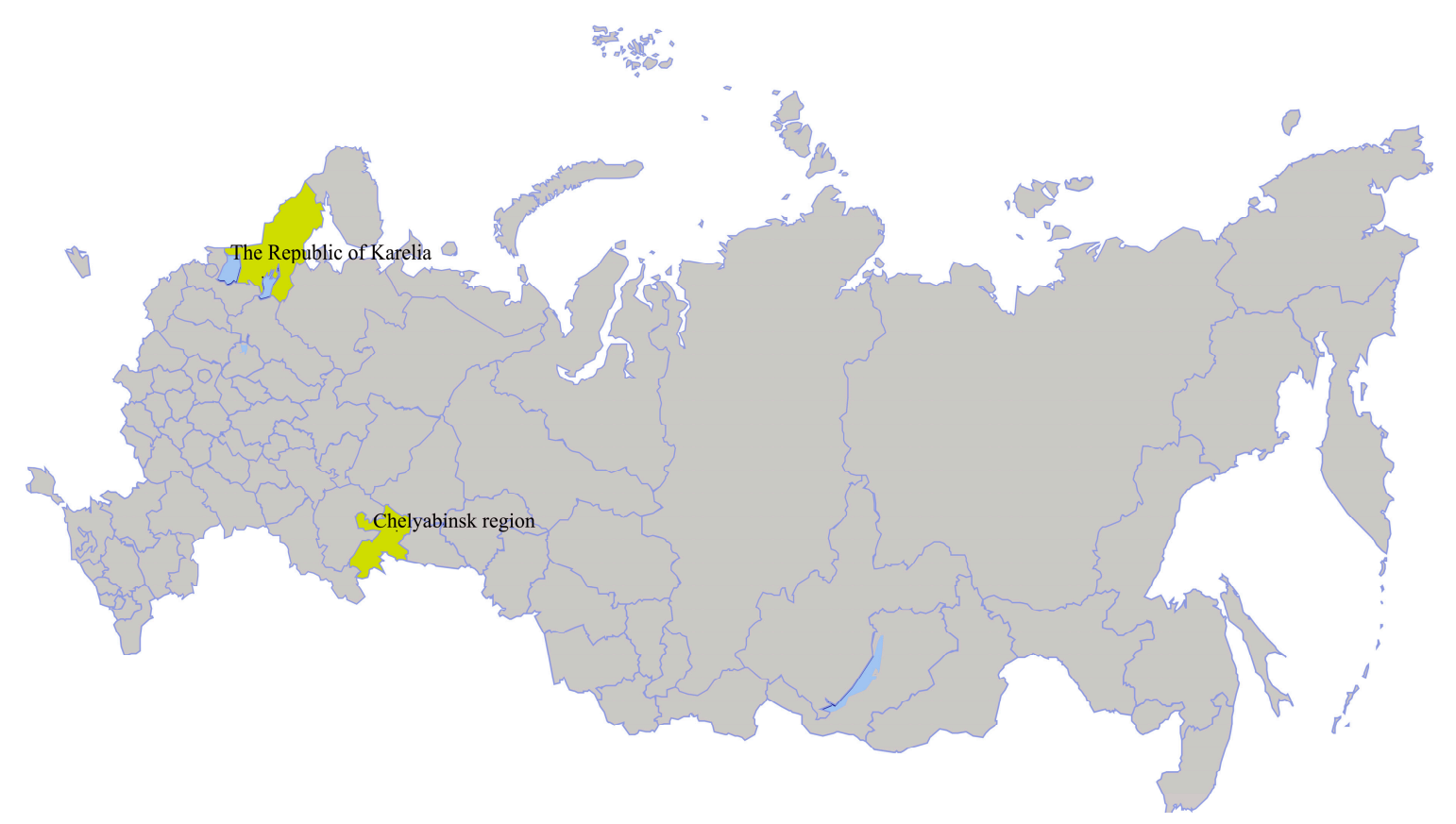

Figure 1. In 2014 from the beginning of May to the middle of June, nymphs and adult Ixodes persulcatus ticks were collected by flagging from vegetation in the Republic of Karelia and the Chelyabinsk region in Russian Federation.

\subsection{Infection of Tick Cell Line}

We used a cell line derived from embryos of the tick I. ricinus-IRE/CTVM19 [22] provided by the Tick Cell Biobank (University of Liverpool, UK). The tick cell line was maintained at $28^{\circ} \mathrm{C}$; in L-15 (Leibovitz) medium (FSBSI Chumakov FSC R\&D IBP RAS, Russia) supplemented with $10 \%$ tryptose phosphate broth, $20 \%$ fetal bovine serum, $2 \mathrm{mM}$ L-glutamine and antibiotics as described earlier [23]. Prior to infection, IRE/CTVM19 cells were seeded in flat-sided culture tubes (Nunc, ThermoFisher Scientific, Waltham, MA, USA) in $2.2 \mathrm{~mL}$ of complete medium and incubated at $28^{\circ} \mathrm{C}$. A week later, cells were infected by adding $200 \mu \mathrm{L}$ of virus-containing material (unfiltered tick homogenate or culture supernate) and incubated at $28{ }^{\circ} \mathrm{C}$;. Medium was changed at fortnightly intervals by removal and replacement of $1.5 \mathrm{~mL}$; the spent medium was used to harvest the virus as described below. Occasional subcultures were carried out at intervals between 1 month and $>1$ year by adding $2.2 \mathrm{~mL}$ of fresh medium, resuspending the tick cells and transferring half of the cell suspension to a new culture tube.

For transmission electron microscopy and high-throughput sequencing, infected cell culture supernate (spent medium) was clarified by centrifugation at $10,000 \mathrm{rpm}$ for $30 \mathrm{~min}$ at $4{ }^{\circ} \mathrm{C}$ using an SW-28 rotor in an Optima L-90K Ultracentrifuge (Beckman Coulter, Brea, CA, USA) and was then ultracentrifuged at 25,000 rpm for $6 \mathrm{~h}$ at $4{ }^{\circ} \mathrm{C}$ using the same rotor. Uninfected cell culture supernate was prepared similarly for transmission electron microscopy.

\subsection{Transmission Electron Microscopy}

The pellet obtained following ultracentrifugation of culture supernate was dissolved overnight in borate saline buffer $\left(0.05 \mathrm{M} \mathrm{H}_{3} \mathrm{BO}_{4}, 0.12 \mathrm{M} \mathrm{NaCl}, 0.24 \mathrm{M} \mathrm{NaOH}\right), \mathrm{pH} 9.0$ (BSB), at $4{ }^{\circ} \mathrm{C}$. A portion of the dissolved pellet was applied to a sucrose density gradient (15-60\%) and centrifuged at 30,000 rpm for $4 \mathrm{~h}$ at $4{ }^{\circ} \mathrm{C}$ using an SW-40 rotor in the Optima L-90K Ultracentrifuge (Beckman Coulter, Brea, CA, USA). To identify resultant density gradient fractions containing virus particles, aliquots of each fraction were subjected to RT-PCR and sequencing of amplified products as described below.

The specimens after ultracentrifugation were adsorbed onto Formvar film attached to 200-mesh nickel EM grids and contrasted with 2\% uranyl acetate [24]. The grids were exposed to UV light for 
one hour to inactivate the virus, air-dried and examined in a JEOL JEM-1400 transmission electron microscope (JEOL, Tokyo, Japan) operated at $80 \mathrm{kV}$. Images were acquired with an Olympus Quemesa digital camera using iTEM software (Olympus Soft Imaging Solutions GmbH, Munster, Germany).

\subsection{Reverse-Transcriptase PCR (RT-PCR) and Sequencing of Amplified Products}

Viral RNA from tick suspensions and culture supernate of infected cells was isolated with TRI Reagent LS (Sigma-Aldrich, St. Louis, MO, USA) according to the manufacturer's protocol. Reverse transcription was performed with random hexamer primers (R6) and M-MLV reverse transcriptase (Promega, Madison, WI, USA) according to the manufacturer's protocol. Viral genomic cDNA was amplified by PCR using specific primers MiassF and MiassR (Table S1). Sequencing was carried out in both directions directly from PCR-amplified DNA on the ABI PRISM 3730 (Applied Biosystems, ThermoFisher Scientific, Waltham, MA, USA) sequencer using ABI PRISM ${ }^{\circledR}$ BigDye $^{\mathrm{TM}}$ Terminator v. 3.1. Genomic sequences were assembled using SeqMan software (DNAstar, Madison, WI, USA).

\subsection{High-Throughput Sequencing}

Total RNA from an ultracentrifugation pellet was fragmented and reverse-transcribed into cDNA with R6 primers using RevertAid reverse transcriptase (ThermoFisher Scientific, Waltham, MA, USA) followed by second strand synthesis with the NEBNext Ultra II Non-Directional RNA Second Strand Synthesis Module (New England Biolabs Inc., Ipswich, MA, USA). Resultant double-stranded DNA was purified using Ampure XP (Beckman Coulter, Brea, CA, USA) and subjected as an input for library preparation process using the NEBNext ${ }^{\circledR}$ Fast DNA Library Prep Set for Ion Torrent ${ }^{\mathrm{TM}}$ (New England Biolabs Inc., Ipswich, MA, USA) following the manufacturer's instructions. The resultant DNA library was quantified with the Ion Library TaqMan ${ }^{\mathrm{TM}}$ Quantitation Kit (ThermoFisher Scientific, Waltham, MA, USA) followed by templating on the Ion Chef instrument (ThermoFisher Scientific, Waltham, MA, USA) and sequencing on the Ion S5XL instrument, with the viral library constituting a part of the Ion 530 Chip. Raw reads were quality-controlled using FaQCs v2.09 [25] and assembled into contigs using SPAdes v3.13.0 [26] with iontorrent flag. The resultant contigs were screened for viral sequences using Blastn algorithm in BLAST v2.9.0+ with nt database and contigs corresponding to virus were extracted for further investigation.

\subsection{Phylogenetic Analysis}

RNA sequences of all published strains of ALSV, representatives of the JMV group and the three strains described in this article were used in the phylogenetic analysis. The nucleotide sequences of the genome coding region of segment 2 were aligned using Clustal-X 2.0.11. Phylogenetic analysis was conducted through the neighbour-joining and the maximum likelihood methods using MEGA 6.0 with 1000 bootstrap replications.

\subsection{Bioinformatics Tools Used for Discovery of Overlapping Elements}

For the analysis we used sequences of the full segment 2 of ALSV, JMTV and Yanggou virus extracted from GenBank (March 12, 2020), as well as sequences of the ALSV strains obtained in the present study (Table S2). Sequences were divided into three groups (named "JMTV", "ALSV" and Yanggou virus) based on their positions in the phylogenetic tree (Figure S1). Sequences obtained in the current study were included in the ALSV alignment.

Analyses of Yanggou virus, ALSV and JMTV were performed separately, because of a low level of similarity between them. Nucleotide sequences of the ORF VP1a (in the case of ALSV and Yanggou virus) and VP1 (in the case of JMTV) were extracted from the full segments. The resultant sequences were codon-aligned using the online tool, Codon Alignment v2.1.0 (available at https: //hcv.lanl.gov/content/sequence/CodonAlign/codonalign.html) with default parameters. The resultant codon alignments were used as an input for the Synplot2 [27] online tool (available at http://guinevere. 
otago.ac.nz/cgi-bin/aef/synplot_c.pl) v. 2014-12-05/03:10:08 with default parameters (no reference sequence, $n=12$ (sliding window of 25 codons)).

\subsection{Genome Annotation and Visualisation}

Putative N-glycosylation sites were predicted using the NetNGlyc 1.0 Server (available online at http://www.cbs.dtu.dk/services/NetNGlyc/) with a standard set of parameters [28]. Transmembrane region prediction was done using the TMHMM Server v. 2.0 (available online at http://www.cbs.dtu. $\mathrm{dk} /$ services/TMHMM/) [29]. Aligned ALSV sequences were examined with the pAliKiss web server (build in June 25 2015, available online at https://bibiserv.cebitec.uni-bielefeld.de/palikiss) with default parameters [30] to find possible conserved RNA structures downstream of putative frameshift sites. ORFs, transmembrane regions and N-glycosylation sites were mapped on the genome using SnapGene Viewer v. 4.3.10. Further image editing was performed in the GNU Image manipulation program v. 2.10 software (https://www.gimp.org).

\section{Results}

\subsection{Detection and Isolation of Alongshan Virus Strains}

We collected 13 nymphs and 156 unfed adult I. persulcatus ticks in Ilmen State Reserve in the Chelyabinsk region and 279 (of which 191 were screened) unfed adult I. persulcatus ticks in Gomselga village in the Republic of Karelia in May-June 2014 (Figure 1). The ticks were pooled (Chelyabinsk region-33 pools, and the Republic of Karelia-45 pools), homogenized and the homogenates screened for flavivirus NS5 RNA. The PCR products from ten flavivirus-positive tick pools were Sanger-sequenced. The resultant sequences were analysed with BLAST. All ten isolates, named Miass501, Miass502, Miass506, Miass508, Miass510, Miass515, Miass519, Miass523, Miass527 and Galozero-14-T20426, were found to have 88.9-92.0\% nucleotide identity to Alongshan virus (Table 1, Table S3).

To isolate the virus from tick homogenates, we inoculated cultures of the IRE/CTVM19 tick cell line. An IRE/CTVM19 culture inoculated with the Miass527 sample was kept for a year with regular medium changes but without passaging or subculture; individual tick cell cultures can be maintained for long periods without subculture [22]. Since the virus did not cause any cytopathic effect in these cells, we changed the medium once every 14 days. Once every 1-2 months we checked the culture medium by RT-PCR for presence of the virus, using specific primers JVsenseV1add-JVasenseV1 and JVsenseV1add-Mi1-2320as (Table S1, Figure S2). After a year of maintenance, $200 \mu \mathrm{L}$ of supernate from the Miass527-infected IRE/CTVM19 culture was passaged into a new flat-sided culture tube with uninfected IRE/CTVM19 cells. This new Miass527-infected culture was subcultured by splitting the cells at a ratio of 1:1 twice in the first 6 months of infection and after that was kept without passaging or subculture. In this condition, Miass527-infected cells have been monitored for over 3 years (observation is still continuing). IRE/CTVM19 cells inoculated with samples Miass519 and Galozero-14-T20426 were each subcultured 1:1 twice in the first 2 months after infection and, at the time of writing, have been monitored for 4 months.

RT-PCR analysis revealed that the strain Miass527 successfully persisted in the tick cell line for a year, followed by one passage and further three years persistence at the time of writing. The other two strains, Miass519 and Galozero-14-T20426, had persisted for at least 4 months.

For two strains, Miass519 (pooled culture supernates collected after one passage at 2 and 3 months after initial isolation) and Miass527 (pooled culture supernates collected after 17, 19, 20 and 32 months after initial isolation), high-throughput sequencing of virus purified from IRE/CTVM19 cell supernate was carried out in order to obtain full genomes, while for the Galozero-14-T20426 strain, partial sequences of segment 2 were obtained via Sanger sequencing using primers JMun1S and Miass_gly_1R (Table 1; Table S1). For the phylogenetic analysis, we used RNA sequences from 18 GenBank entries of representatives of the JMV group and the three ALSV strains isolated in the present study (Table 1). 
The phylogenetic analysis was performed on a 1,732 bp fragment of segment 2 using MEGA 6.0 with the neighbour-joining method. Interestingly, one of the JMTV isolates (MN095520-isolate JMTV/I.ricinus/France) clustered with the ALSV sequences. For consistency, we included this JMTV sequence in all our analyses of ALSV (see below). All three of our strains belonged to ALSV and formed one monophyletic group with the strain H3 isolated in China (Figure 2). When using the maximum likelihood method, the phylogenetic tree structure was not changed.

Table 1. Alongshan virus strains isolated in unfed adult Ixodes persulcatus ticks from two geographically separate regions of Russia.

\begin{tabular}{|c|c|c|c|}
\hline Strain & Tick Species & Year, Region (GPS) & GenBank Access. No. \\
\hline Miass519 & I. persulcatus & \multirow{2}{*}{$\begin{array}{c}\text { 2014, Chelyabinsk region, } \\
\text { Ilmen State Reserve } \\
\left(55^{\circ} 01.287^{\prime} \mathrm{N} 060^{\circ} 10.097^{\prime} \mathrm{E}\right)\end{array}$} & MN648774-MN648777 \\
\hline Miass527 & I. persulcatus & & MN648770-MN648773 \\
\hline Galozero-14-T20426 & I. persulcatus & $\begin{array}{l}\text { 2014, The Republic of Karelia } \\
\left(62^{\circ} 4.515^{\prime} \mathrm{N} 33^{\circ} 57.077^{\prime} \mathrm{E}\right)\end{array}$ & MN604229 \\
\hline
\end{tabular}

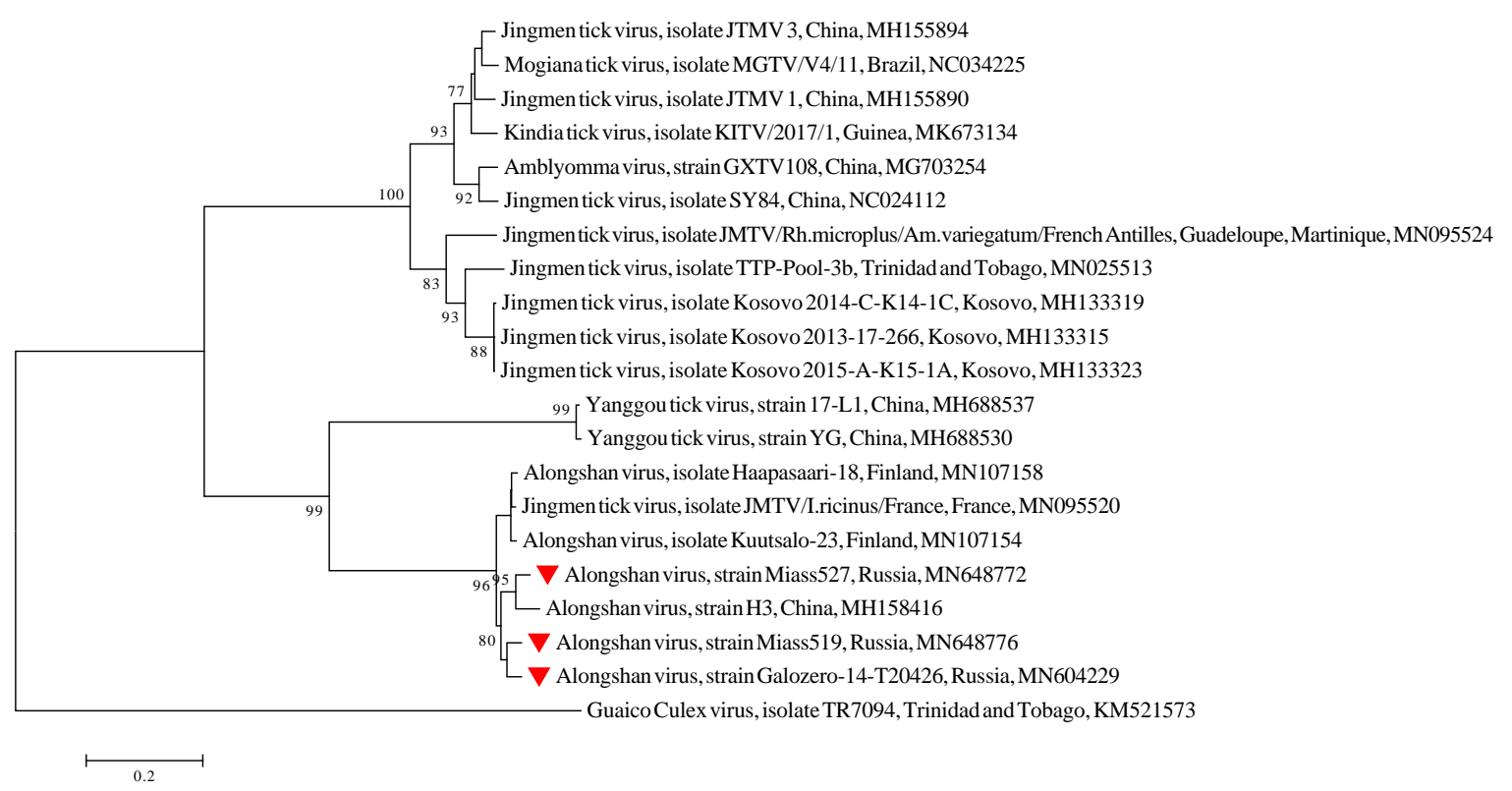

Figure 2. Phylogenetic tree of a 1,732 bp fragment of segment 2 of Alongshan virus and other members of the Jingmenvirus group. Phylogenetic trees were constructed using MEGA 6.0 with the neighbour-joining method (1000 bootstrap replications). Bootstrap values ( $>70 \%)$ are shown at the branches. GenBank accession numbers are listed for each strain. $\mathbf{\nabla}$ Strains from the present study.

\subsection{Transmission Electron Microscopy}

We used pools of cell culture supernate collected after one passage of strain Miass527 in IRE/CTVM19 cells, at 17, 19, 20 and 32 months after initial isolation for transmission electron microscopic examination. The IRE/CTVM19 tick cell line was recently reported to contain RNA sequences with similarity to rhabdoviruses [31]. To prevent confusion between ALSV and this putative rhabdovirus in our microscopic examination, we first screened the pellet obtained after ultracentrifugation of uninfected IRE/CTVM19 for rhabdovirus RNA using specific primers (Table S1). We detected an unknown rhabdovirus and named it IRE/CTVM19-associated rhabdovirus, strain BSLab (GenBank accession number-MT181988). Since this new rhabdovirus was also detected in the Miass527-infected IRE/CTVM19 cell pellet, we subjected the dissolved pellet to sucrose density gradient separation and selected the fraction with the largest amount of ALSV RNA and very low levels of rhabdovirus RNA (Figure 3). 


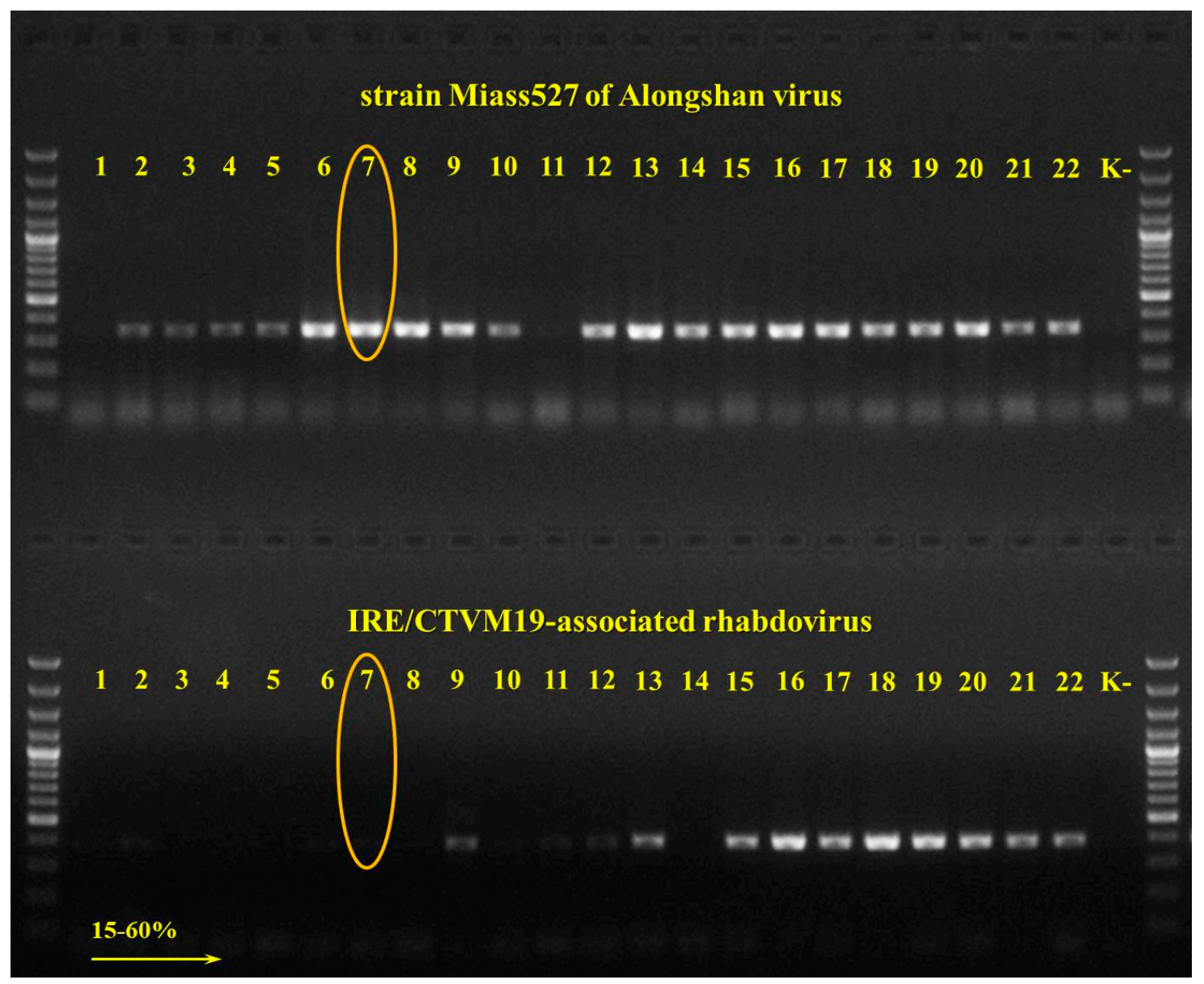

Figure 3. Electrophoresis of PCR fragments of sucrose density gradient fractions of Alongshan virus-infected IRE/CTVM19 cells. We separated Alongshan virus (ALSV) strain Miass527 and IRE/CTVM19-associated rhabdovirus strain BSLab by sucrose density gradient centrifugation to identify the fraction with the largest amount of ALSV RNA. Viral genomic cDNA was amplified by PCR using specific primers for ALSV (Miass_gly_3F and Miass_gly_3R) and IRE/CTVM19-associated rhabdovirus (Rhabdo_L_1F and Rhabdo_L_1R). Fraction \#7 was selected for transmission electron microscopy. \#\#1-22-gradient fractions, K- negative control.

The microscopy revealed that most virions of the strain Miass527 were enveloped spherical particles with a diameter of $40.5 \pm 3.7 \mathrm{~nm}$ (Figure 4A,B; Figure S3). They had either electrontranslucent or electron-dense cores. The viral particles with an electron-dense core could have lost genomic RNA (Figure 4B). In the same gradient fraction, we found small spherical particles with a diameter of $13.1 \pm 2.1 \mathrm{~nm}$, which had electron-translucent or electron-dense cores (Figure 4C,D; Figure S4). These small particles could be virions with incomplete genome, protein structures or some other unidentified component of IRE/CTVM19 cells. The pellet obtained after ultracentrifugation of uninfected IRE/CTVM19 culture supernate was separated by sucrose density gradient ultracentrifugation. For transmission electron microscopy, we used the same fraction of the gradient as used for the ALSV-infected IRE/CTVM19 cells. In this fraction we found small spherical particles with a diameter of $15.7 \pm 1.8 \mathrm{~nm}$, which had electron-dense cores (Figure 4E; Figure S5). These small particles could be virions of an unknown virus, protein structures or some other unidentified component of IRE/CTVM19 cells. No particles resembling rhabdoviruses were seen in gradient fraction \#7 (Figure 3) from either ALSV-infected or uninfected IRE/CTVM19 cells. 

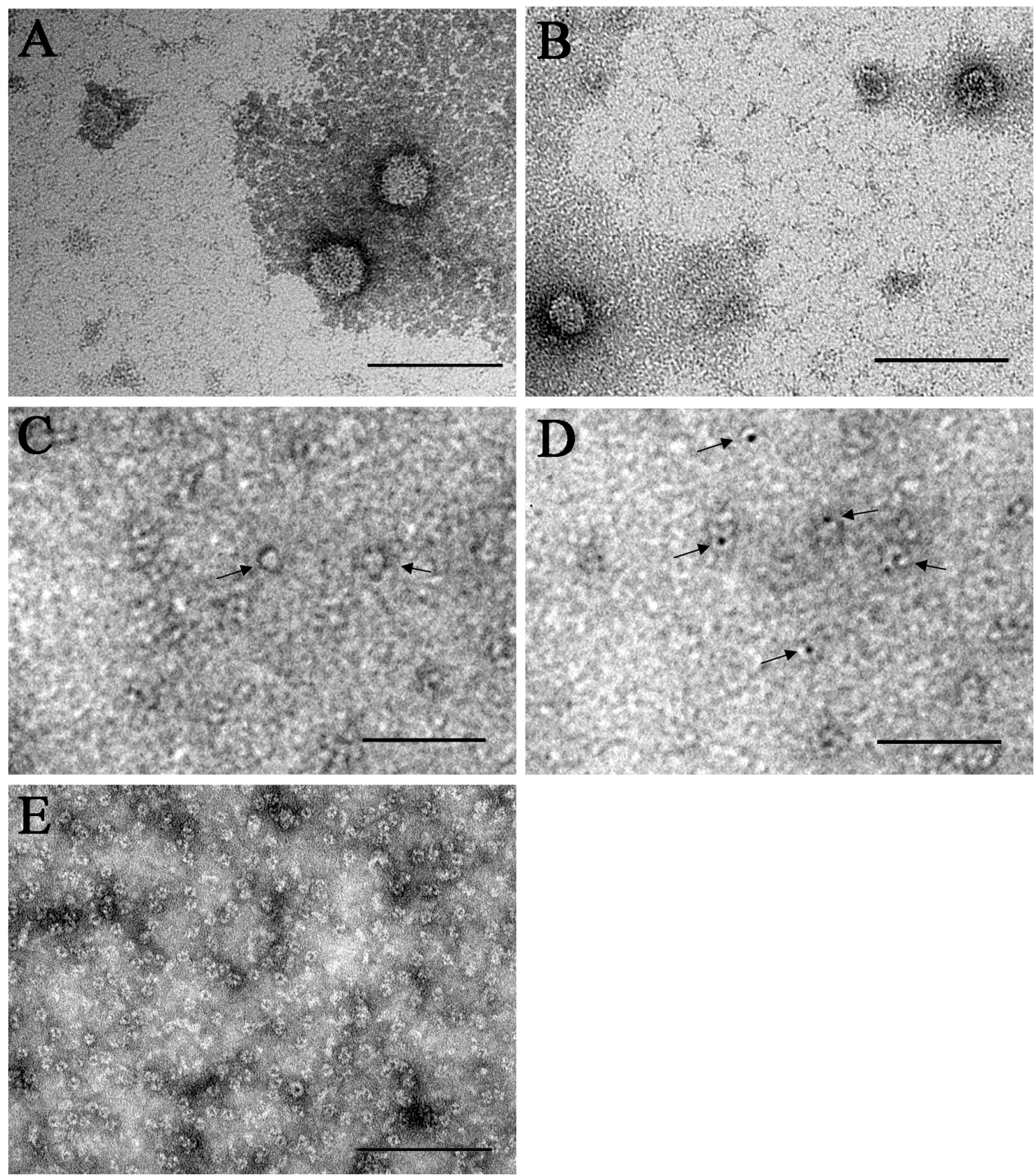

Figure 4. Transmission electron microscopy. Electron micrographs of purified viral particles of the Alongshan virus strain Miass527 propagated in IRE/CTVM19 cells (A,B). Small particles with electron-translucent (C) or electron-dense (D) cores were seen. Small particles with electron-dense cores were also seen in the equivalent fraction of ultracentrifuged supernate from uninfected IRE/CTVM19 cells (E). Samples were stained with 2\% uranyl acetate. Scale bars, $100 \mathrm{~nm}$.

\subsection{Full Genome, Genome Structure and Proposed Novel Elements}

We sequenced full genomes of the strains Miass527 and Miass519. Segments 1, 3 and 4 of the newly-isolated Miass strains were more similar to each other than to previously-described ALSV strains. However, segment 2 of the strain Miass527 was more similar to the Chinese strain H3 than to Miass519, while segment 2 of the strain Miass519 was closer to JMTV/I.ricinus/France than to Miass527 (Table 2). These data suggest that reassortment of genome segments within ALSV could occur. Similar observations were made earlier for JMTV [4]. 
Table 2. Percentage nucleotide identity of the four genome segments of different strains of Alongshan virus for which full genomes were available at the time of writing. Genbank accession numbers for each strain and isolate are as follows: Miass519 (MN648774-MN648777) (this study); Miass527 (MN648770-MN648773) (this study); H3 (MH158415-MH158418) [5]; Kuutsalo-23 (MN107153-MN107155) and Haapasaari-18 (MN107157-MN107160) [12]; JMTV/I.ricinus/France (MN095519-MN095522) [8].

\begin{tabular}{|c|c|c|c|c|c|}
\hline & $\begin{array}{c}\text { Strain } \\
\text { Miass527 }\end{array}$ & Strain $\mathrm{H} 3$ & $\begin{array}{c}\text { Strain } \\
\text { Kuutsalo-23 }\end{array}$ & $\begin{array}{c}\text { Strain } \\
\text { Haapasaari-18 }\end{array}$ & $\begin{array}{c}\text { Isolate } \\
\text { JMTV/I.ricinus/France }\end{array}$ \\
\hline \multicolumn{6}{|c|}{ Segment 1 . NS5-like protein } \\
\hline Strain Miass519 & $90.6 \%$ & $89.0 \%$ & $89.2 \%$ & $88.9 \%$ & $89.1 \%$ \\
\hline Strain Miass527 & & $89.6 \%$ & $89.2 \%$ & $88.6 \%$ & $89.7 \%$ \\
\hline Strain H3 & & & $89.6 \%$ & $89.2 \%$ & $90.1 \%$ \\
\hline Strain Kuutsalo-23 & & & & $95.6 \%$ & $96.0 \%$ \\
\hline Strain Haapasaari-18 & & & & & $95.9 \%$ \\
\hline \multicolumn{6}{|c|}{ Segment 2. VP1a and VP1b } \\
\hline Strain Miass519 & $92.6 \%$ & $92.0 \%$ & $93.7 \%$ & $93.6 \%$ & $94.0 \%$ \\
\hline Strain Miass527 & & $94.7 \%$ & $92.4 \%$ & $92.2 \%$ & $93.0 \%$ \\
\hline Strain H3 & & & $91.7 \%$ & $91.6 \%$ & $92.1 \%$ \\
\hline Strain Kuutsalo-23 & & & & $98.3 \%$ & $98.5 \%$ \\
\hline Strain Haapasaari-18 & & & & & $98.6 \%$ \\
\hline \multicolumn{6}{|c|}{ Segment 3. NS3-like protein } \\
\hline Strain Miass519 & $96.7 \%$ & $91.2 \%$ & $91.3 \%$ & $90.9 \%$ & $91.2 \%$ \\
\hline Strain Miass527 & & $90.7 \%$ & $91.1 \%$ & $90.5 \%$ & $91.0 \%$ \\
\hline Strain H3 & & & $90.9 \%$ & $91.3 \%$ & $91.5 \%$ \\
\hline Strain Kuutsalo-23 & & & & $95.0 \%$ & $97.4 \%$ \\
\hline Strain Haapasaari-18 & & & & & $94.5 \%$ \\
\hline \multicolumn{6}{|c|}{ Segment 4 . VP2 and VP3 } \\
\hline Strain Miass519 & $98.6 \%$ & $90.5 \%$ & $90.8 \%$ & $90.7 \%$ & $91.2 \%$ \\
\hline Strain Miass527 & & $90.6 \%$ & $90.7 \%$ & $90.8 \%$ & $91.3 \%$ \\
\hline Strain H3 & & & $90.6 \%$ & $90.3 \%$ & $91.1 \%$ \\
\hline Strain Kuutsalo-23 & & & & $94.7 \%$ & $98.0 \%$ \\
\hline Strain Haapasaari-18 & & & & & $95.3 \%$ \\
\hline
\end{tabular}

Overall, the full genomes of the Miass strains demonstrated $>88 \%$ nucleotide identity to the other strains of ALSV known to date, with glycoprotein-coding segment 2 having the highest similarity and NS5-like protein coding segment 1 being the most divergent.

The two Miass strains were similar in their genome organisation to each other and to known ALSV. Segments 1 and 3 were monocistronic. Segment 1 contained a flavivirus-NS5-like protein with polymerase and putative methyltransferase domains and a predicted signal peptide on the $\mathrm{N}$-terminus of the protein. Segment 3 contained a flavivirus NS3-like protein with proposed functions of serine protease and RNA helicase (Figure 5). Both proteins had transmembrane regions corresponding to the previously-mapped strain H3. Segment 4 contained two non-flavivirus-related ORFs. There was a slippery sequence (G GTT TTT) and some evidence of an RNA-structured region (Figure S6) and we believe that the VP3 ORF may be expressed via -1 ribosome frameshifting (Figure 5), as was previously shown for other ALSV strains. Regarding the VP1a and VP1b ORFs in segment 2, transmembrane regions and $\mathrm{N}$-glycosylation sites were also in positions in the predicted amino acid sequences similar to the other ALSV strains [5]. Interestingly, we were not able to identify signal peptides in either VP2 or VP1a using the TMHMM v.2.0 software. 

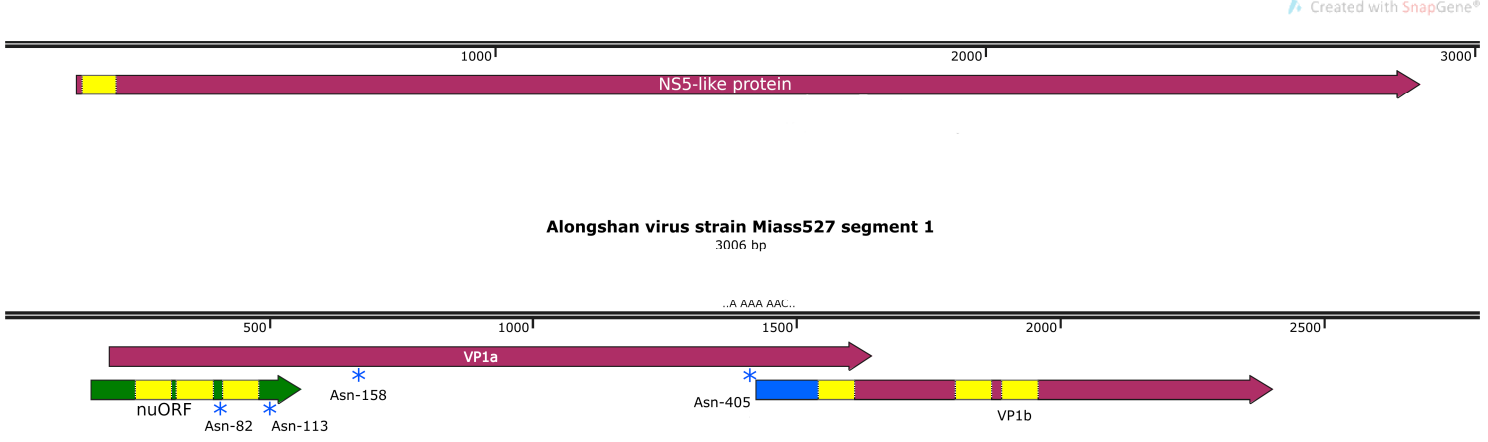

Alongshan virus strain Miass527 segment 2
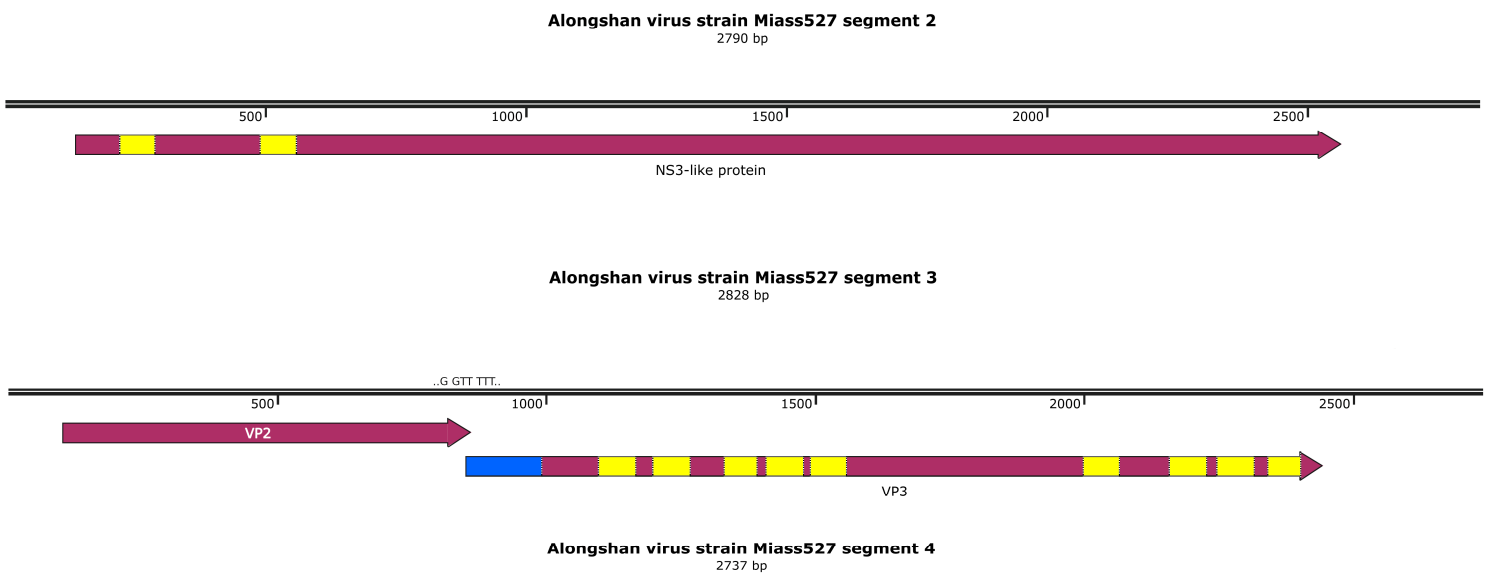

Figure 5. Genome of the Alongshan virus strain Miass527. Transmembrane regions are shown in yellow, the part of the VP1b protein derived from the proposed ribosomal frameshift is shown in blue, and the proposed novel protein in segment 2 is in green. Blue stars mark the putative N-glycosylation sites.

We performed a search of the functional elements in the VP1a region with the Synplot2 program [27]. Using codon alignment of four previously-described ALSV strains (MH158416, MN107154, MN107158, MN095520) and the three strains sequenced in this study, we discovered two regions of high conservation in the VP1a ORF (Figure 6A). The first one corresponded to the $3^{\prime}$-terminal region of the ORF. We believe that such a high level of conservation in this region was caused by an overlap with the VP1b ORF. We managed to find a slippery motif (A AAA AAC) followed by a spacer and an RNA structure (Figure S7) in this region of the VP1a ORF that theoretically would allow VP1b to be translated via -1 ribosome frameshift (Figure 5).

Additionally, Synplot2 allowed us to identify an unusually high conservation rate in the first $\sim 110$ codons of VP1a ORF in segment 2 . We believe that this conservation was caused by an additional ORF that was found in this region. This novel upstream ORF (nuORF) is $399 \mathrm{nt}$ long and has a $365 \mathrm{nt}$ overlap with the VP1a ORF (Figure 6A). 

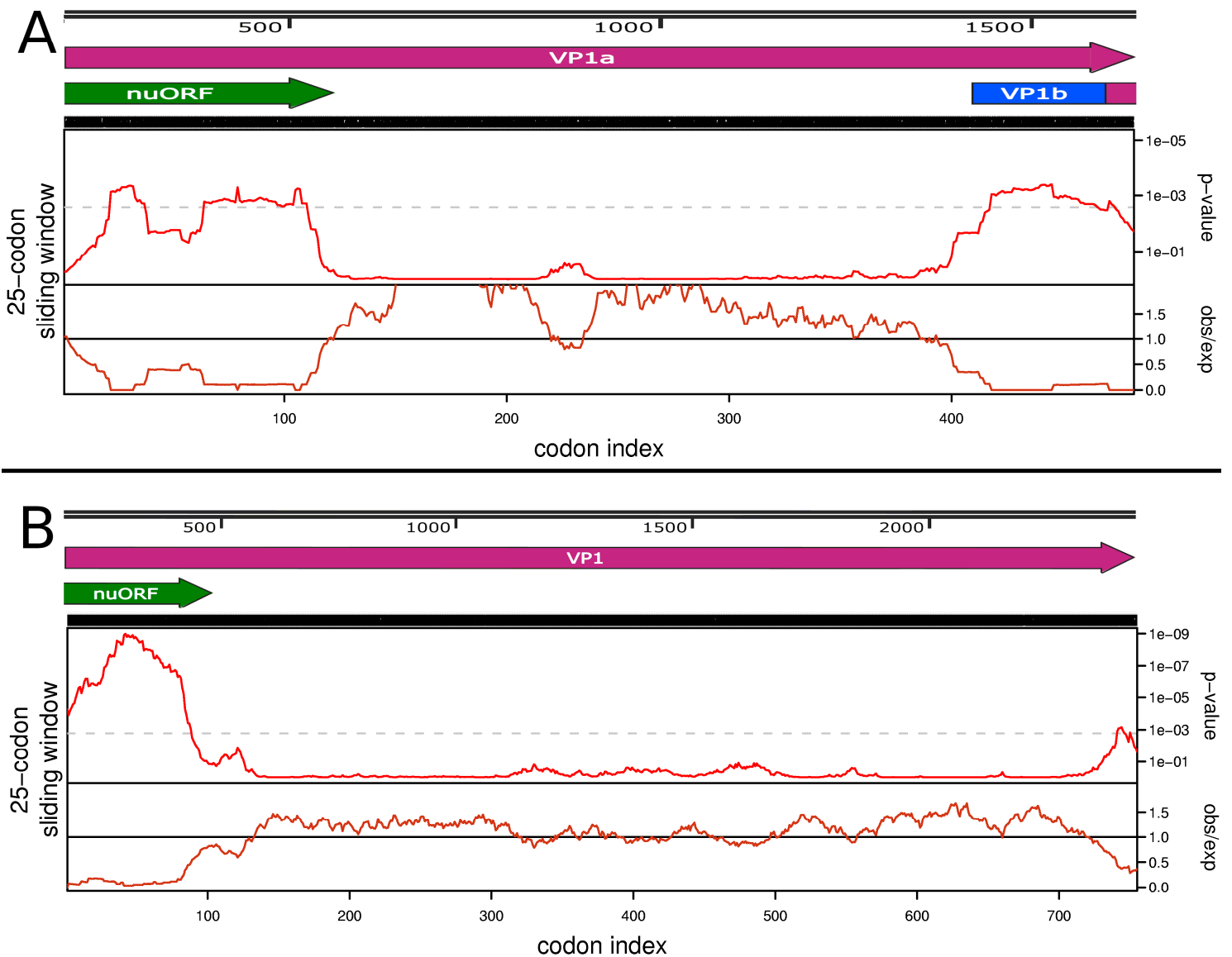

Figure 6. Association of the novel upstream open reading frame (nuORF) with the region of high conservation in the Alongshan virus VP1a and Jingmen tick virus VP1 open reading frames. (A) Codon alignment of 7 full sequences of the Alongshan virus VP1a open reading frame (ORF) was used in the analysis (Table S2). (B) Codon alignment of all 25 full sequences of the Jingmen tick virus VP1 ORF was used in the analysis (Table S2). In each section, the top panel represents the virus genome map of the tested region. The middle panel depicts the probability that the degree of ORF conservation within a 25-codon sliding window could be obtained under neutral evolution. The grey dashed line indicates $p=0.005$ significance (after correcting for multiple tests, where the number of tests is the length of a coding sequence divided by the window size). The bottom panel displays the relative amount of synonymous-site conservation at a 25 -codon sliding window by showing the ratio of the observed number of synonymous substitutions to the expected number. The analysis was done using the Synplot2 program [27].

Application of the tblastn algorithm using the protein sequence encoded by the Miass527 nuORF as an entry revealed homologs within the JMV group (Table S2). Upon further inspection, all of the resultant sequences were shown to have a nuORF. Thus, we decided to test known JMTV sequences for the existence of the additional ORF upstream of the VP1 region with Synplot2.

We searched for a full-length segment 2 of JMTV using blast algorithm and strain SY84 JMTV full segment 2 sequence as an entry. Since relationships within the JMV group are not yet decided and some representatives of this group have different names, we could not use a GenBank search. Sequences MN095532 and KX377514 were found to have unknown nucleotides in the middle of the segment and were not used in the analysis. For these sequences, nuORF was confirmed to be intact. We then constructed a phylogenetic tree (Figure S1) that allowed us to identify three groups (Jingmen tick, Yanggou and Alongshan viruses) that should be analysed separately due to phylogenetic distance. All JMTV sequences with full segment 2 (25 entries) known to date (March 12, 2020) have a nuORF. We performed analysis of the JMTV segment 2 sequences (Figure 6B) and Synplot2 showed 
high conservation in the first $\sim 100$-codon region. This demonstrates that the region associated with nuORF is conserved more highly than would be expected under neutral evolution in both ALSV and JMTV sequences.

We also tested the Yanggou virus sequences and, although all of the full genome sequences had a nuORF, the Synplot2 program did not find a region of higher-than-expected conservation in the first 100 codons of Yanggou virus VP1a (Figure S8). However, there are only three known full-length segment 2 sequences of Yanggou virus and the number of sequences is important for acquiring statistics needed for a prediction.

Overall, the ALSV nuORF encoded 132 amino acids. According to the prediction, this protein may have an N-terminal signal sequence and three transmembrane regions with a C-terminal tail, thus making the nuORF product a small membrane protein (Figure 7A).
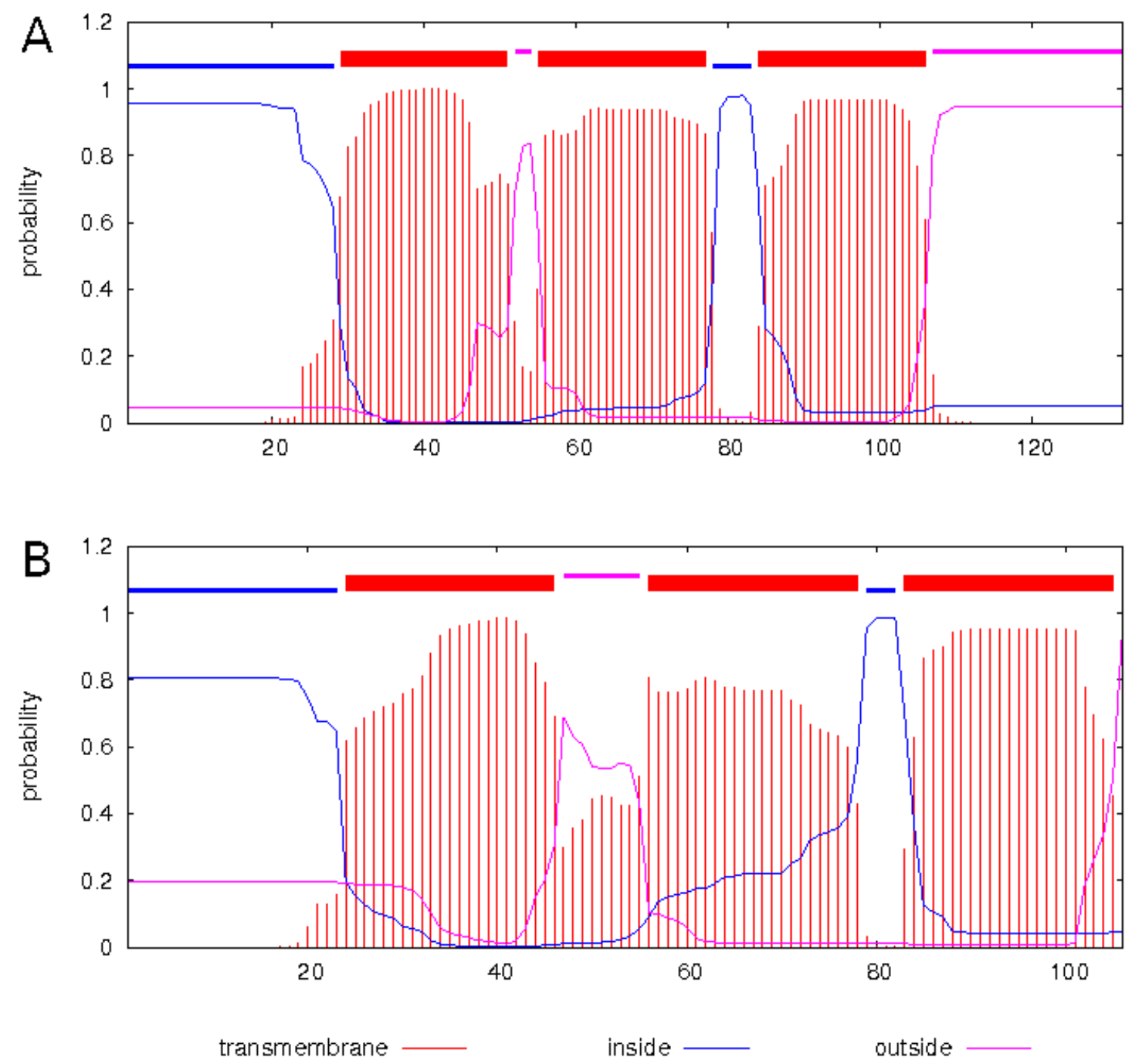

Figure 7. Transmembrane domains of the proposed nuORF product of (A) Alongshan virus strain Miass527, (B) Jingmen tick virus strain SY84. The analysis was done using TMHMM Server v. 2.0 program [29].

The predicted protein encoded by the nuORF of JMTVs was shorter ( 106 aa) and had $~ 50 \%$ identity to the ALSV nuORF product. It had an overall structure similar to that of ALSV, with a proposed N-terminal signal sequence and three predicted transmembrane regions, but, in contrast to the ALSV nuORF, it had no C-terminal tail (Figure 7B). 


\section{Discussion}

Due to advances in high-throughput sequencing and viral metagenomics, many new flavi-like viruses have been discovered in recent decades [1,4,13]. A large number of flavi-like viruses have been detected in or isolated from arthropods [1,32,33], but their ability to cause infection and disease in vertebrates and their medical significance are not yet known.

The JMV group is a recently-discovered group of segmented ssRNA(+) viruses that infect different species of invertebrates, including insects, ticks and nematodes [1,4,9,12,15-17]. Moreover, some representatives of the new flavi-like viruses were detected in patients who had other severe infectious diseases [11], and these viruses might themselves cause illness in humans [5,9]. There is still little information about the replication and overall coding strategy of this novel virus group.

ALSV is a representative of the JMV group. It was first isolated in China from a patient suffering fever of unknown aetiology [5]. Subsequently, the virus was detected in I. persulcatus ticks and C. tritaeniorhynchus and A. yatsushiroensis mosquitoes in China [5] and in I. ricinus ticks in Finland [12] and France [8]. It was shown that ALSV is able to infect mammals and cause illness in humans (with symptoms including headache, fever, fatigue and depression) [5,18]. Thus, additional isolates of this virus from new geographical locations will be important for understanding its distribution, as well as for the local healthcare systems. New genome data could also provide additional information on the structural organisation of the ALSV genome.

We detected ALSV in nine pools of I. persulcatus ticks collected in Chelyabinsk region in the south of European Russia, and in one pool from the Republic of Karelia in north-west Russia. ALSV was isolated from three of the ten positive pools into IRE/CTVM19 cells. We sequenced two full genomes of the new ALSV strains Miass527 and Miass519 from Chelyabinsk region and obtained partial sequences of segment 2 for the ALSV strain Galozero-14-T20426 from the Republic of Karelia. Although these three strains were isolated from territories distant from each other, phylogenetic analysis of the segment 2 sequences showed that they form a single monophyletic group with the strain H3 isolated in China.

Chinese strains of ALSV were isolated from mammalian blood samples using Vero (African green monkey) cells $[5,18]$ while the isolation of the virus detected in Finnish ticks failed in Vero and two human cell lines [12]. The Chinese JMTV strain isolated from A. javanense ticks replicated successfully in the $R$. microplus embryo-derived tick cell line BME/CTVM23 but failed to grow in Vero, DH82 (canine monocyte-macrophage-derived cells) and C6/36 (Aedes albopictus mosquito) cells [9]. We isolated three tick-derived strains of ALSV using the tick cell line IRE/CTVM19; our results, together with those of the previous studies reporting successful in vitro isolation and continuous propagation of JMV group viruses $[5,9,18]$, suggest the possibility that mammal-derived viruses will more readily infect mammalian cells, while tick-derived viruses may be more infective for tick cells. However, additional isolations of JMV group viruses will be required to test this theory and the possible underlying genetic causes. For the first time we showed that the ALSV strain Miass527 can persist in tick cells for at least three years. It was previously shown that the tick cell line IRE/CTVM19 may harbor an unknown rhabdovirus [31]. Our experiment confirmed the presence of a rhabdovirus and suggests that it does not interfere with the persistence and active reproduction of ALSV in IRE/CTVM19 cells. Persistent infection in tick cells and ticks could affect virus properties, as was previously shown for tick-borne encephalitis virus [34].

Previously, it was shown that the virion sizes of JMV group representatives ranged from 30-35 nm to 70-100 nm [2,4,5]. ALSV virions were reported to be enveloped spherical (or nearly spherical) particles with a diameter of approximately 80-100 nm [5]. The size of virions of our strain Miass527 was $40.5 \pm 3.7 \mathrm{~nm}$. In transmission electron microscopy, some of the viral particles had electron-dense cores, and could hypothetically be defective virions without genomic RNA [35]. In addition, we found smaller particles with a diameter of $13.1 \pm 2.1 \mathrm{~nm}$, which had either electron-translucent or electron-dense cores. Presumably, they could represent either: (a) glycoprotein aggregates, similar to the "classic" flavivirus nonstructural protein 1, which is secreted from infected cells [36,37], (b) protein structures appearing after long-term virus persistence, (c) virions with incomplete genome, or (d) virions of an 
unknown virus or other unknown structures present in IRE/CTVM19 cells. In the equivalent gradient fraction of uninfected cells, we found only particles with a diameter of $15.7 \pm 1.8 \mathrm{~nm}$, i.e., slightly larger than the small particles that we saw in the same fraction of Miass527-infected cells. In any case, the particles were smaller than the smallest parvovirus virions with a diameter of 18-26 nm [38]. Further investigations are needed to clarify the nature of these particles.

Although the strain Miass527 of ALSV did not differ in overall genome structure from the previously isolated ALSV [5], the newly-obtained data allowed us to propose a novel protein encoded by both ALSV and JMTV and, probably, by some other members of the JMV group. The nuORF, proposed here, is conserved in all the full segment 2 sequences of ALSV, JMTV and Yanggou virus available at the time of writing. Considering that ALSV and JMTV are somewhat distantly related (around 70\% nucleotide identity and around 80\% amino acid identity in the NS5-like protein), it is likely that the nuORF indeed encodes a functional virus protein. Nevertheless, further studies are needed to confirm its expression and function during the virus life cycle.

Interestingly, an ORF of a more distant member of the JMV group, the mosquito-borne Guaico Culex virus [2], was discovered in the same genome region. It also encodes a small protein, but with no amino acid identity to the nuORF. A mass spectrometry analysis of the Guaico Culex virus virion proteins did not identify any peptides that belong to this putative ORF, thus making its product (if it is indeed expressed) a nonstructural protein [2].

If the nuORF product protein is indeed functional at some point during ALSV replication, it would most likely be a minor membrane protein. Such proteins are present in a variety of RNA viruses and perform different functions. For example, in flaviviruses, small membrane proteins NS2B and NS4A (with sizes similar to the nuORF) perform multiple functions, ranging from enzyme cofactors $[39,40]$ to participation in particle formation $[41,42]$ and protection against the cellular antiviral response. Influenza A M2 protein acts as a proton-selective ion channel [43], whereas Coronavirus E protein acts as a viroporin and plays a role in induction of the regulation of the unfolded protein response [44]. Nevertheless, confirmation of the nuORF expression and understanding of its possible functions should be subjects for further study.

\section{Conclusions}

We isolated three strains of ALSV from I. persulcatus ticks in Russia. Although these strains were isolated from territories far from each other, in phylogenetic analysis they formed a single monophyletic group with the virus isolated in China. Our study confirmed the value of tick cell lines in virus isolation and maintenance of persistent infection. Availability of these novel ALSV strains has enabled better understanding of the phylogenetic relationships within the JMV group, and will facilitate further study of the origins, structure, replication, epidemiology, transmission, vertebrate hosts and medical and veterinary significance of these enigmatic segmented RNA viruses.

Supplementary Materials: The following are available online at http://www.mdpi.com/1999-4915/12/4/362/s1, Table S1: Specific primers for amplification of genome segments 1 and 2 of Alongshan virus and IRE/CTVM19associated rhabdovirus; Table S2: List of Jingmenvirus group sequences used for the discovery of the conservative elements within segment 2; Table S3: Additional Alongshan virus isolates from Chelyabinsk region detected by screening of tick pools using a heminested RT-PCR for the flavivirus NS5 gene; Figure S1: Phylogenetic tree of all full segment 2 sequences of the Jingmenvirus group; Figure S2: Results of the RT-PCR at selected time points of Alongshan virus strain Miass527 persistence in the IRE/CTVM19 tick cell line; Figure S3: The range of virion size detected in transmission electron microscopy of strain Miass527 of Alongshan virus; Figure S4: The size of the small spherical particles detected in transmission electron microscopy of strain Miass527 of Alongshan virus; Figure S5: The size of the small spherical particles detected in transmission electron microscopy of ultracentrifuged supernate from uninfected IRE/CTVM19 cells; Figure S6: Predicted RNA structure downstream of the frameshift region in segment 4 of Alongshan viruses (ALSV); Figure S7: Predicted RNA structure downstream of the frameshift region in segment 2 of Alongshan viruses (ALSV); Figure S8: Synonymous site conservation analysis of the Yanggou virus VP1a ORF. 
Author Contributions: Conceptualisation, G.G.K. and A.P.G.; methodology, I.S.K. and A.G.L.; software, A.G.L. and A.M.S.; validation, I.S.K., A.G.L., O.A.B., G.G.K. and A.G.L.; formal analysis, A.G.L.; investigation, I.S.K., A.G.L., A.S.K., O.A.B., A.E.P., A.Y.I., A.S.Y., S.V.B., L.A.B., A.M.S. and L.V.G.; resources, S.V.L., L.B.-S., V.A.G., N.A.N., A.P.G. and G.G.K.; data curation, I.S.K. and A.G.L.; writing-original draft preparation, I.S.K., A.G.L., O.A.B., L.B.-S. and G.G.K.; writing-review and editing, I.S.K., A.G.L., O.A.B., L.B.-S. and G.G.K.; visualisation, I.S.K., A.G.L. and N.A.N.; supervision, A.P.G. and G.G.K.; project administration, I.S.K., A.G.L. and G.G.K.; funding acquisition, G.G.K., L.B.-S., S.V.B. and V.A.G. All authors have read and agreed to the published version of the manuscript.

Funding: Tick collection by Sergey V. Bugmyrin and Liubov A. Bespyatova is supported by the state order (project № 0218-2019-0075). Lesley Bell-Sakyi is supported by the United Kingdom Biotechnology and Biological Sciences Research Council grant number BB/P024270/1.

Conflicts of Interest: The authors declare no conflict of interest.

\section{References}

1. Shi, M.; Lin, X.-D.; Vasilakis, N.; Tian, J.-H.; Li, C.-X.; Chen, L.-J.; Eastwood, G.; Diao, X.-N.; Chen, M.-H.; Chen, X.; et al. Divergent Viruses Discovered in Arthropods and Vertebrates Revise the Evolutionary History of the Flaviviridae and Related Viruses. J. Virol. 2016, 90, 659-669. [CrossRef] [PubMed]

2. Ladner, J.T.; Wiley, M.R.; Beitzel, B.; Auguste, A.J.; Dupuis, A.P.; Lindquist, M.E.; Sibley, S.D.; Kota, K.P.; Fetterer, D.; Eastwood, G.; et al. A Multicomponent Animal Virus Isolated from Mosquitoes. Cell Host Microbe 2016, 20, 357-367. [CrossRef] [PubMed]

3. Maruyama, S.R.; Castro-Jorge, L.A.; Ribeiro, J.M.C.; Gardinassi, L.G.; Garcia, G.R.; Brandão, L.G.; Rodrigues, A.R.; Okada, M.I.; Abrão, E.P.; Ferreira, B.R.; et al. Characterisation of divergent flavivirus NS3 and NS5 protein sequences detected in Rhipicephalus microplus ticks from Brazil. Mem. Inst. Oswaldo Cruz 2014, 109, 38-50. [CrossRef] [PubMed]

4. Qin, X.-C.; Shi, M.; Tian, J.-H.; Lin, X.-D.; Gao, D.-Y.; He, J.-R.; Wang, J.-B.; Li, C.-X.; Kang, Y.-J.; Yu, B.; et al. A tick-borne segmented RNA virus contains genome segments derived from unsegmented viral ancestors. Proc. Natl. Acad. Sci. USA 2014, 111, 6744-6749. [CrossRef] [PubMed]

5. Wang, Z.-D.; Wang, B.; Wei, F.; Han, S.-Z.; Zhang, L.; Yang, Z.-T.; Yan, Y.; Lv, X.-L.; Li, L.; Wang, S.-C.; et al. A New Segmented Virus Associated with Human Febrile Illness in China. N. Engl. J. Med. 2019, 380, 2116-2125. [CrossRef] [PubMed]

6. Lefkowitz, E.J.; Dempsey, D.M.; Hendrickson, R.C.; Orton, R.J.; Siddell, S.G.; Smith, D.B. Virus taxonomy: The database of the International Committee on Taxonomy of Viruses (ICTV). Nucleic Acids Res. 2018, 46, D708-D717. [CrossRef]

7. Yong, C.Y.; Yeap, S.K.; Omar, A.R.; Tan, W.S. Advances in the study of nodavirus. PeerJ 2017, 5, e3841. [CrossRef]

8. Temmam, S.; Bigot, T.; Chrétien, D.; Gondard, M.; Pérot, P.; Pommelet, V.; Dufou, E.; Petres, S.; Devillers, E.; Hoem, T.; et al. Insights into the Host Range, Genetic Diversity, and Geographical Distribution of Jingmenviruses. mSphere 2019, 4, 1-13. [CrossRef]

9. Jia, N.; Liu, H.B.; Ni, X.B.; Bell-Sakyi, L.; Zheng, Y.C.; Song, J.L.; Li, J.; Jiang, B.G.; Wang, Q.; Sun, Y.; et al. Emergence of human infection with Jingmen tick virus in China: A retrospective study. EBioMedicine 2019, 43, 317-324. [CrossRef]

10. Dinçer, E.; Hacıoğlu, S.; Kar, S.; Emanet, N.; Brinkmann, A.; Nitsche, A.; Özkul, A.; Linton, Y.-M.; Ergünay, K. Survey and Characterization of Jingmen Tick Virus Variants. Viruses 2019, 11, 1071. [CrossRef]

11. Emmerich, P.; Jakupi, X.; von Possel, R.; Berisha, L.; Halili, B.; Günther, S.; Cadar, D.; Ahmeti, S.; Schmidt-Chanasit, J. Viral metagenomics, genetic and evolutionary characteristics of Crimean-Congo hemorrhagic fever orthonairovirus in humans, Kosovo. Infect. Genet. Evol. 2018, 65, 6-11. [CrossRef] [PubMed]

12. Kuivanen, S.; Levanov, L.; Kareinen, L.; Sironen, T.; Jääskeläinen, A.J.; Plyusnin, I.; Zakham, F. Detection of novel tick-borne pathogen, Alongshan virus, in Ixodes ricinus ticks, south-eastern Finland. Eurosurveillance 2019, 24, 1900394. [CrossRef] [PubMed]

13. Villa, E.C.; Maruyama, S.R.; de Miranda-Santos, I.K.F.; Palacios, G.; Ladner, J.T. Complete Coding Genome Sequence for Mogiana Tick Virus, a Jingmenvirus Isolated from Ticks in Brazil. Genome Announc. 2017, 5 , 17-18. [CrossRef] [PubMed] 
14. de Oliveira Pascoal, J.; de Siqueira, S.M.; da Costa Maia, R.; Szabó, M.P.J.; Yokosawa, J. Detection and molecular characterization of Mogiana tick virus (MGTV) in Rhipicephalus microplus collected from cattle in a savannah area, Uberlândia, Brazil. Ticks Tick. Borne. Dis. 2019, 10, 162-165. [CrossRef] [PubMed]

15. Webster, C.L.; Waldron, F.M.; Robertson, S.; Crowson, D.; Ferrari, G.; Quintana, J.F.; Brouqui, J.M.; Bayne, E.H.; Longdon, B.; Buck, A.H.; et al. The discovery, distribution, and evolution of viruses associated with Drosophila melanogaster. Plos Biol. 2015, 13, e1002210. [CrossRef] [PubMed]

16. Callister, D.M.; Winter, A.D.; Page, A.P.; Maizels, R.M. Four abundant novel transcript genes from Toxocara canis with unrelated coding sequences share untranslated region tracts implicated in the control of gene expression. Mol. Biochem. Parasitol. 2008, 162, 60-70. [CrossRef]

17. Tetteh, K.K.A.; Loukas, A.; Tripp, C.; Maizels, R.M. Identification of abundantly expressed novel and conserved genes from the infective larval stage of Toxocara canis by an expressed sequence tag strategy. Infect. Immun. 1999, 67, 4771-4779. [CrossRef]

18. Wang, Z.-D.; Wang, W.; Wang, N.N.; Qiu, K.; Zhang, X.; Tana, G.; Liu, Q. Prevalence of the emerging novel Alongshan virus infection in sheep and cattle in Inner Mongolia, northeastern China. Parasit. Vectors 2019, 12, 450. [CrossRef]

19. Filippova, N.A. Ixodid Ticks of the Subfamily Ixodinae. Fauna of the USSR: Arachnoides; Nauka: Leningrad, Russia, 1977; Volume 4.

20. Filippova, N.A. Fauna of Russia and neighbouring countries. Ixodid ticks of Subfamily Amblyomminae. Arachnoidea; Nauka: Saint Petersburg, Russia, 1997; Volume 4.

21. Scaramozzino, N.; Crance, J.-M.; Jouan, A.; DeBriel, D.A.; Stoll, F.; Garin, D. Comparison of Flavivirus universal primer pairs and development of a rapid, highly sensitive heminested reverse transcription-PCR assay for detection of flaviviruses targeted to a conserved region of the NS5 gene sequences. J. Clin. Microbiol. 2001, 39, 1922-1927. [CrossRef]

22. Bell-Sakyi, L.; Zweygarth, E.; Blouin, E.F.; Gould, E.A.; Jongejan, F. Tick cell lines: Tools for tick and tick-borne disease research. Trends Parasitol. 2007, 23, 450-457. [CrossRef]

23. Weisheit, S.; Villar, M.; Tykalová, H.; Popara, M.; Loecherbach, J.; Watson, M.; Růžek, D.; Grubhoffer, L.; De La Fuente, J.; Fazakerley, J.K.; et al. Ixodes scapularis and Ixodes ricinus tick cell lines respond to infection with tick-borne encephalitis virus: Transcriptomic and proteomic analysis. Parasites Vectors 2015, 8, 599. [CrossRef] [PubMed]

24. Nikitin, N.; Trifonova, E.; Evtushenko, E.; Kirpichnikov, M.; Atabekov, J.; Karpova, O. Comparative study of non-enveloped icosahedral viruses size. PLOS ONE 2015, 10, e0142415. [CrossRef]

25. Lo, C.C.; Chain, P.S.G. Rapid evaluation and quality control of next generation sequencing data with FaQCs. BMC Bioinform. 2014, 15, 366. [CrossRef] [PubMed]

26. Bankevich, A.; Nurk, S.; Antipov, D.; Gurevich, A.A.; Dvorkin, M.; Kulikov, A.S.; Lesin, V.M.; Nikolenko, S.I.; Pham, S.; Prjibelski, A.D.; et al. SPAdes: A new genome assembly algorithm and its applications to single-cell sequencing. J. Comput. Biol. 2012, 19, 455-477. [CrossRef] [PubMed]

27. Firth, A.E. Mapping overlapping functional elements embedded within the protein-coding regions of RNA viruses. Nucleic Acids Res. 2014, 42, 12425-12439. [CrossRef] [PubMed]

28. Gupta, R.; Jung, E.; Brunak, S. Prediction of N-glycosylation sites in human proteins. 2004. Available online: http://www.cbs.dtu.dk/services/NetNGlyc/ (accessed on 25 March 2020).

29. Krogh, A.; Larsson, È.; Von Heijne, G.; Sonnhammer, E.L.L. Predicting Transmembrane Protein Topology with a Hidden Markov Model: Application to Complete Genomes. J. Mol. Biol. 2001, 305, 567-580. [CrossRef]

30. Janssen, S.; Giegerich, R. The RNA shapes studio. Bioinformatics 2015, 31, 423-425. [CrossRef]

31. Bell-Sakyi, L.; Attoui, H. Virus discovery using tick cell lines. Evol. Bioinforma. 2016, 12, 31-34.

32. Huhtamo, E.; Putkuri, N.; Kurkela, S.; Manni, T.; Vaheri, A.; Vapalahti, O.; Uzcategui, N.Y. Characterization of a Novel Flavivirus from Mosquitoes in Northern Europe That Is Related to Mosquito-Borne Flaviviruses of the Tropics. J. Virol. 2009, 83, 9532-9540. [CrossRef]

33. Huhtamo, E.; Cook, S.; Moureau, G.; Uzcátegui, N.Y.; Sironen, T.; Kuivanen, S.; Putkuri, N.; Kurkela, S.; Harbach, R.E.; Firth, A.E.; et al. Novel flaviviruses from mosquitoes: Mosquito-specific evolutionary lineages within the phylogenetic group of mosquito-borne flaviviruses. Virology 2014, 464-465, 320-329. [CrossRef]

34. Belova, O.A.; Litov, A.G.; Kholodilov, I.S.; Kozlovskaya, L.I.; Bell-Sakyi, L.; Romanova, L.I.; Karganova, G.G. Properties of the tick-borne encephalitis virus population during persistent infection of ixodid ticks and tick cell lines. Ticks Tick. Borne. Dis. 2017, 8, 895-906. [CrossRef] [PubMed] 
35. Korboukh, V.K.; Lee, C.A.; Acevedo, A.; Vignuzzi, M.; Xiao, Y.; Arnold, J.J.; Hemperly, S.; Graci, J.D.; August, A.; Andino, R.; et al. RNA Virus Population Diversity: An Optimum for Maximal Fitness and Virulence. J. Biol. Chem. 2014, 289, 29531-29544. [CrossRef] [PubMed]

36. Alcalá, A.C.; Medina, F.; González-Robles, A.; Salazar-Villatoro, L.; Fragoso-Soriano, R.J.; Vásquez, C.; Cervantes-Salazar, M.; del Angel, R.M.; Ludert, J.E. The dengue virus non-structural protein 1 (NS1) is secreted efficiently from infected mosquito cells. Virology 2016, 488, 278-287. [CrossRef] [PubMed]

37. Muller, D.A.; Landsberg, M.J.; Bletchly, C.; Rothnagel, R.; Waddington, L.; Hankamer, B.; Young, P.R. Structure of the dengue virus glycoprotein non-structural protein 1 by electron microscopy and single-particle analysis. J. Gen. Virol. 2012, 93, 771-779. [CrossRef] [PubMed]

38. Mi, X.; Bromley, E.; Joshi, P.U.; Long, F.; Heldt, C.L. Virus isoelectric point determination using single-particle chemical force microscopy. Langmuir 2019. [CrossRef]

39. Lindenbach, B.D.; Rice, C.M. Molecular biology of flaviviruses. Adv. Virus Res. 2003, 59, $23-61$.

40. Shiryaev, S.A.; Chernov, A.V.; Aleshin, A.E.; Shiryaeva, T.N.; Strongin, A.Y. NS4A regulates the ATPase activity of the NS3 helicase: A novel cofactor role of the non-structural protein NS4A from West Nile virus. J. Gen. Virol. 2009, 90, 2081-2085. [CrossRef]

41. Muñoz-Jordan, J.L.; Sánchez-Burgos, G.G.; Laurent-Rolle, M.; García-Sastre, A. Inhibition of interferon signaling by dengue virus. Proc. Natl. Acad. Sci. USA 2003, 100, 14333-14338. [CrossRef]

42. Li, X.; Deng, C.; Ye, H.; Zhang, H.; Zhang, Q.; Chen, D.; Zhang, P.; Shi, P.-Y.; Yuan, Z.-M.; Zhang, B. Transmembrane Domains of NS2B Contribute to both Viral RNA Replication and Particle Formation in Japanese Encephalitis Virus. J. Virol. 2016, 90, 5735-5749. [CrossRef]

43. DiMaio, D. Viral Miniproteins. Annu. Rev. Microbiol. 2014, 68, 21-43. [CrossRef]

44. Schoeman, D.; Fielding, B.C. Coronavirus envelope protein: Current knowledge. Virol. J. 2019, 16, 69. [CrossRef] [PubMed]

(C) 2020 by the authors. Licensee MDPI, Basel, Switzerland. This article is an open access article distributed under the terms and conditions of the Creative Commons Attribution (CC BY) license (http://creativecommons.org/licenses/by/4.0/). 